1. Improve symptom management.

2. Reduce medicine administration errors.

3. Release $\mathrm{RN}$ time to care.

Method

- Literature review and pre- training questionnaire.

- SNAD group formed.

- Networking with other hospices.

- Create teaching package, provide study day including competency assessment.

- Devised competency tool and Standard Operating Procedure (SOP).

Results Pre-SNAD training timings were recorded with an average administration of medicine taking 15 minutes. The study day was evaluated well with RNs feeling empowered and confident. Evaluation of the project hasn't taken place yet, as competency is ongoing, however, preliminary findings show more efficient symptom management, less interruptions and increased autonomy. We are hopeful that we will see a reduction in medication errors because of this. We have trained $75 \%$ of the RNs, those not trained do not meet the inclusion criteria but will be trained later in the year.

Conclusion Already we are finding that $\mathrm{RN}$ work has been interrupted less during the SNAD process. Medications have been administered more promptly therefore patients' symptoms managed more efficiently. By utilising SNAD, time is used more efficiently in an environment that has a small number of staff and so limited workforce resilience.

\section{P-141 ANTICIPATORY PRESCRIBING AT END-OF-LIFE: DO WE NEED TO CHANGE PRACTICE?}

Theresa Tran, Charlotte Lee, Joy Ross. St Christopher's Hospice, London, UK

10.1136/spcare-2021-Hospice.158

Background/Aims Anticipatory prescribing (AP) of injectable medications at the end-of-life for community patients is good practice to achieve timely symptom control (National Institute for Health and Care Excellence. Care of dying adults in the last days of life, 2015). However robust evidence to support current practice is lacking. We evaluated baseline AP practices at a South London hospice against current local guidelines.

Methods This retrospective audit looked at patient case notes and anticipatory charts (June-August 2020) using a standardised proforma including: drug choice, dose, indication and subsequent patient clinical trajectory. Anticipatory drug use, drug wastage and themes from case vignettes are presented.

Results 76 patients were reviewed, median age 80 (41-107), $66 \%$ male. $52 \%$ had a cancer diagnosis. All patients were prescribed and dispensed four medications for: pain, agitation, secretions and nausea/vomiting. There was close adherence to local guidelines (choice of drug, dose). Most commonly used drugs were: morphine 61\%, midazolam 99\%, glycopyrronium $97 \%$ and haloperidol $88 \%$. 94\% of patients died within three months (median 9 days). Eleven patients required admission to hospital or hospice.

$64 \%$ had stats given at end-of-life, 53\% for pain, $41 \%$ for agitation, $24 \%$ for secretions; only $16 \%$ needed an antiemetic. We saw wide variation in prescribing practices for seizures at end-of-life (11 patients). Multiple health care professionals (Clinical Nurse Specialists, District Nurses and the
London Ambulance Service) administered stats in and out of hours. Further training is required to ensure appropriate dose escalation and titration. Dispensing all four medications costs approximately $£ 50$ per patient; haloperidol alone accounts for $3 / 5$ ths of that cost and is not often used.

Conclusions Few patients required all four medications, where anti-emetics are needed the choice of drug warrants further review with likely cost savings. At least $50 \%$ of our medications could be dispensed by GPs rather than hospice pharmacy. Further education/training will improve individualised AP and tailored administration of medications at endof-life, including improved confidence around seizure management. We are currently updating our guidelines to reflect this.

\section{DRUG INSTRUCTION CHART COMPLETION - IMPROVING OUR SERVICE DURING COVID-19}

Simone Ali, Leila Donald, Evelyn Prodger. Martlets Hospice, Hove, UK

\subsection{6/spcare-2021-Hospice. 159}

Introduction As part of discussions at the Nurse Independent Prescriber Group, we agreed to focus on the team's use of Drug Instruction Charts because:

- They are widely used for end of life care prescribing.

- They are used in an anticipatory way.

- They are used by staff outside of our service to initiate treatment.

- There were particular challenges during COVID-19 due to some prescribers working remotely; historically, our charts have been completed by hand.

Method We developed an audit tool with two parts:

Part 1 - Completion of form - all patient details complete; legibility; additional information documented (e.g. estimated glomerular filtration rate [eGFR], transdermal medication also in use).

Part 2 - Appropriateness of prescribing - drugs prescribed were appropriate in line with diagnosis, prognosis, risk, renal function.

The audit was carried out by the head of community services and an associate specialist/consultant in palliative medicine.

We also created a process to enable remote completion of the Drug Instruction Charts. This was far from simple, involving many staff with IT skills and access to additional software (and a determined Medical Director!). A Standard Operating Procedure for electronic completion of Drug Instruction Charts was developed to ensure robust governance.

Results Results were input into a spreadsheet developed by the hospice data analyst. Each prescriber submitted Drug Instruction Charts they had completed. Results were shared with each individual prescriber for their learning. Overall results were shared with all prescribers.

Conclusions Compliance with the form and appropriateness of prescribing were overwhelmingly positive but there are improvements that can be made to practice. The process of developing the electronic chart was in itself helpful as it opened up discussions about electronic completion of ReSPECT (Recommended Summary Plan for Emergency Care and Treatment) form, as well as governance. The implementation of electronic completion of the charts means that 